\title{
Rotation Measures of Extragalactic Sources Behind the Southern Galactic Plane: New Insights into the Large-Scale Magnetic Field of the Inner Milky Way
}

\author{
J. C. Brown, ${ }^{1}$ M. Haverkorn, ${ }^{2,3}$ B. M. Gaensler, ${ }^{4,5,6}$ A. R. Taylor, ${ }^{1}$ N. S. Bizunok,${ }^{5}$ \\ N. M. McClure-Griffiths, ${ }^{7}$ J. M. Dickey ${ }^{8}$ and A. J. Green ${ }^{4}$
}

\begin{abstract}
We present new Faraday rotation measures (RMs) for 148 extragalactic radio sources behind the southern Galactic plane $\left(253^{\circ} \leq \ell \leq 356^{\circ},|b| \leq 1.5^{\circ}\right)$, and use these data in combination with published data to probe the large-scale structure of the Milky Way's magnetic field. We show that the magnitudes of these RMs oscillate with longitude in a manner that correlates with the locations of the Galactic spiral arms. The observed pattern in RMs requires the presence of at least one large-scale magnetic reversal in the fourth Galactic quadrant, located between the Sagittarius-Carina and Scutum-Crux spiral arms. To quantitatively compare our measurements to other recent studies, we consider all available extragalactic and pulsar RMs in the region we have surveyed, and jointly fit these data to simple models in which the large-scale field follows the spiral arms. In the best-fitting model, the magnetic field in the fourth Galactic quadrant is directed clockwise in the Sagittarius-Carina spiral arm (as viewed from the North Galactic pole), but is oriented counter-clockwise in the Scutum-Crux arm. This contrasts with recent analyses of pulsar RMs alone, in which the fourth-quadrant field was presumed to be directed counter-clockwise in the Sagittarius-Carina arm. Also in contrast to recent pulsar RM studies, our joint modeling of pulsar and extragalactic RMs demonstrates that large numbers of large-scale magnetic field reversals are not required to account for observations.
\end{abstract}

Subject headings: Galaxy: structure — ISM: magnetic fields — polarization

\footnotetext{
${ }^{1}$ Department of Physics and Astronomy, University of Calgary, T2N 1N4, Canada; jocat@ras.ucalgary.ca; russ@ras.ucalgary.ca

${ }^{2}$ Jansky Fellow, National Radio Astronomy Observatory

${ }^{3}$ Astronomy Department, UC-Berkeley, 601 Campbell Hall, Berkeley, CA 94720; marijke@astro.berkeley.edu

${ }^{4}$ School of Physics A29, The University of Sydney, NSW 2006, Australia; bgaensler@usyd.edu.au; agreen@physics.usyd.edu.au

${ }^{5}$ Harvard-Smithsonian Center for Astrophysics, 60 Garden Street, Cambridge, MA 01238; nbizunok@cfa.harvard.edu

${ }^{6}$ Alfred P. Sloan Research Fellow, Australian Research Council Federation Fellow

${ }^{7}$ Australia Telescope National Facility, CSIRO, PO Box 76, Epping, NSW 1710, Australia; Naomi.McClureGriffiths@csiro.au

${ }^{8}$ Physics Department, University of Tasmania, Private Bag 21, Hobart TAS 7001, Australia;
}

\section{Introduction}

The Galactic magnetic field is now recognized as a fundamental component of the interstellar medium, and plays a critical role in the formation and evolution of structures in the Milky Way. An important prediction in models of the large-scale magnetic field, in both the Milky Way and in other galaxies, is the existence of magnetic field reversals (regions of magnetic shear across which the field changes direction by roughly $180^{\circ}$ ). Determining the number and location of these magnetic reversals is essential to understanding Galactic evolution (Shukurov 2005). While the majority of the recent studies suggest several large-scale reversals in the Galaxy along its radius, it is interesting to

John.Dickey@utas.edu.au 
note that most other galaxies exhibit either one reversal or none (Beck 2007). Is our Galaxy unique this way, or is it simply a difference in observing methods?

The large-scale Galactic magnetic field is concentrated in the disk, and is most often studied via observations of rotation measure (RM), the measurable consequence of Faraday rotation. For a source that emits linearly polarised radiation at an angle $\phi_{\circ}$, the received radiation will have a polarisation angle at a wavelength, $\lambda[\mathrm{m}]$, given by:

$$
\phi=\phi_{\circ}+\lambda^{2} 0.812 \int n_{e} \mathbf{B} \cdot \mathbf{d l}=\phi_{\circ}+\lambda^{2} \mathrm{RM}
$$

where $n_{e}\left[\mathrm{~cm}^{-3}\right]$ is the electron density and $\mathbf{B}[\mu \mathrm{G}]$ is the magnetic field along the propagation path dl $[\mathrm{pc}]$. The RM integral is over the path from the source to the observer.

For more than four decades, the RMs of both pulsars and extragalactic radio sources (EGS) have been used to probe the Galactic magnetic field. This has led to a series of clear conclusions. First, in the local spiral arm, the field is unquestionably directed clockwise $(\mathrm{CW})$, as viewed from the North Galactic pole (Manchester 1972, 1974; Heiles 1996a). The field in the first quadrant (Q1; $0^{\circ} \leq \ell \leq 90^{\circ}$; see Figure 1) of the Sagittarius-Carina spiral arm is reversed relative to the local arm (Thomson \& Nelson 1980; Simard-Normandin \& Kronberg 1980; Lvne \& Smith 1989), implying a large-scale field reversal between the local arm and the Q1 component of the Sagittarius-Carina arm. Some evidence suggests that this reversed field extends into the fourthquadrant $\left(\mathrm{Q} 4 ; 270^{\circ} \leq \ell \leq 360^{\circ}\right)$ component of the Sagittarius-Carina arm (eg. Rand \& Lyne 1994; Han et al. 1999; Frick et al. 2001).

At larger distances, the existence of other largescale reversals in the Galaxy remains unclear. Ideally, reconstruction of the Galactic magnetic field should utilize information from both pulsars and extragalactic sources. However, until recently there have been very few EGS RMs available at low-latitude that might be used to study the field in the disk. Studies that have utilized pulsar RMs alone are constrained by the comparatively sparse sampling of pulsars on the sky, which can make it difficult to map the field in complicated regions.

Three recent pulsar RM studies are as follows. Using pulsar RMs, Weisberg et al. (2004) inves- tigated the existence and location of reversals in Q1, concluding that a reversal occurs between each arm so that the magnetic fields in adjacent arms are oppositely directed. While the evidence presented by Weisberg et al. (2004) for a reversal between the local arm and the Sagittarius-Carina arm in Q1 is indisputable, their evidence for additional reversals is based on limited data, and they acknowledge that the evidence for a reversal into the Q4-component of the Sagittarius-Carina arm is not well-defined.

Vallée (2005) investigated azimuthal field configurations in the Galaxy using pulsar RMs averaged in concentric rings. He concluded that bestfit for this model was an overall clockwise magnetic field with a $2 \mathrm{kpc}$ wide counter-clockwise (CCW) ring, located between 4 and $6 \mathrm{kpc}$ from the Galactic center (note that a non-standard Solar-circle radius of $7.2 \mathrm{kpc}$ was assumed in this study). In this model, the Galactic field has two large-scale reversals, and the Q1 component of the Sagittarius-Carina arm is CCW, while the Q4 component is $\mathrm{CW}$.

Using 223 new pulsar RM observations primarily in the fourth quadrant, in conjunction with previous pulsar RMs, Han et al. (2006) concluded there is a reversal at every arm-interarm boundary, so that the fields in the arms are directed CCW, and the interarm regions are directed CW. A potential inconsistency with this model is that the majority of the pulsars distributed along the Q4 component of the Sagittarius-Carina spiral arm have what Han et al. (2006) describe as 'unexpectedly positive' RMs, which they suggest is the influence of HII regions along the lines-of-sight to the affected pulsars (see also Mitra et al. 2003).

Recent surveys of the Galactic plane at high resolution and at multiple wavelengths have assisted greatly in the study of the Galactic magnetic field by addressing the previous paucity of low-latitude EGS RM data (eg. Brown \& Taylor 2001). One such survey is the Southern Galactic Plane Survey (SGPS; McClure-Griffiths et al. 2005; Haverkorn et al. 2006b). The SGPS images low latitudes in the third and fourth quadrants of the Galaxy, complementing the Canadian Galactic Plane Survey in the northern hemisphere (CGPS; Taylor et al. 2003). Rotation measures calculated from these data were used by Haverkorn et al. (2006a) to explore the structure of the small-scale 
field. Here, we present the tabulation of these RMs and use them to examine the structure of the large-scale field.

\section{Rotation Measure Calculations}

The initial set of observations for the SGPS (ie. Phase I) spans an area of $253^{\circ} \leq \ell \leq$ $358^{\circ}$ and $|b| \leq 1.5^{\circ}$. The observations were done with the Australia Telescope Compact Array (ATCA) in New South Wales, Australia. For details about the SGPS observations and polarimetric data reduction, see Gaensler et al. (2001) and Haverkorn et al. (2006b).

RMs were calculated using twelve separate 8 $\mathrm{MHz}$ bands centered on frequencies between 1336 $\mathrm{MHz}$ and $1432 \mathrm{MHz}$. The proximity of the 8 $\mathrm{MHz}$ bands allows for unambiguous RM calculations using the algorithm designed by Brown et al. (2003b) for the CGPS, with appropriate modifications for the ATCA. Specifically, an RM calculation was considered reliable if the source was sufficiently polarized $(>0.3 \%)$, had sufficient signal to noise $(>2)$ across at least 3 pixels, was Faraday thin (Vallée 1980), and had a consistent value across the source.

Of the 215 polarised source candidates identified, 148 sources had RMs that successfully passed the screening tests discussed above. These new data are given in Table 1 . Three of our 148 sources had a RM determined by Gaensler et al. (2001), as part of the SGPS test region $\left(325.5^{\circ} \leq \ell \leq\right.$ $\left.332.5^{\circ},-0.5 \leq b \leq 3.5^{\circ}\right)$. The previously determined values of these sources are within the errors of the new values quoted in Table 1 . These sources are indicated by footnote marker $d$. Two additional test region RM sources fall within the latitude range of the SGPS but they lie within the noise perimeter of our data (see Gaensler et al. 2001) and were not observable. There is one other previously observed RM from an EGS, reported by Broten et al. (1988), that falls within our field at $\ell=307.1^{\circ}, b=1.2^{\circ}$. This source is resolved in the SGPS and exhibits a large gradient across the source. Consequently, it failed the screening, though its calculated RM $\left(+183 \pm 23 \mathrm{rad} \mathrm{m}^{-2}\right)$ is in agreement with the previously determined value $\left(+185 \pm 1 \mathrm{rad} \mathrm{m}^{-2}\right)$.

\section{Observations of the Galactic Magnetic Field}

Figure 2 shows the RMs of EGS and pulsars in the SGPS region. Most of the pulsars (99 of 120) are from Han et al. (2006). The remaining are from Taylor et al. (2000) and Han et al. (1999). The most striking feature of Figure 2 is the change in sign of RM from predominantly positive at low longitudes to predominantly negative at high longitudes for both the pulsars and EGS at $\ell \sim 304^{\circ}$, though it is more prominent for the EGS. A change in sign of the overall trend in RM can only come from a change of direction in the dominant lineof-sight component of the magnetic field. The fact that the RM sign change is abrupt indicates that this directional change is the result of a physical field reversal, rather than a viewing angle effect such as that observed towards $\ell \sim 180^{\circ}$ (eg. Brown \& Tavlor 2001). Furthermore, the abruptness indicates a thin current sheet and an associated large gradient in the magnetic field (Heiles 1996b).

By averaging the EGS RMs in $\ell$ across the sky to reduce small-scale variations (for example, due to the small-scale field or intrinsic effects from the EGS themselves), we can obtain more information about the large-scale field. The top panel in Figure 3 shows the RM data from the SGPS, both raw and binned plotted as a function of Galactic longitude. The middle panel shows these data smoothed. In all three presentations of the EGS data, an oscillating pattern of RM with longitude is visible. The transition from positive to negative RMs remains at $\ell \sim 304^{\circ}$, indicated by the solid vertical line. In the bottom panel of Figure 3. we plot the individual pulsar RMs as a function of Galactic longitude. These data were not averaged in $\ell$ because of the variation and uncertainty in the pulsar distances. In spite of this, there are features seen here similar to the oscillatory pattern observed in the EGS data.

The dashed and dotted lines in the middle panel of Figure 3 are the approximate longitudes of $|\mathrm{RM}|$ maxima and minima respectively for the SGPS data. In Figure 1, we show these lines as lines-ofsight overlaid on a top-down view of the Galaxy where the grey scale is the Galactic electron density model of Cordes \& Lazio (2002), hereafter CL02. Interestingly, the blue dashed lines $(|\mathrm{RM}|$ 
maxima) tend to have the longest continuous fraction of their length along a spiral arm or through the central annulus put in the CL02 to correspond to the molecular ring. This is consistent with the expectation that EGS RMs should be dominated by the spiral arms as a result of the higher electron densities in these regions (Han et al. 2006). The strong positive RMs in the longitude range around $\ell \sim 292^{\circ}$, seen in both the EGS and pulsars, suggest the magnetic field in the Q4-component of the Sagittarius-Carina arm is directed CW. The same conclusion was reached by Caswell et al. (2004) using data from a distant supernova remnant. This CW field presents a simple explanation for the positive pulsar RMs identified by Han et al. (2006) as part of a 'Carina anomaly'.

Similarly, the strong negative RMs in the longitude range around $\ell \sim 312^{\circ}$ suggest the magnetic field in the Q4-component of the ScutumCrux arm is directed CCW. Therefore, a magnetic field reversal must reside between the SagittariusCarina arm and the Scutum-Crux arm in Q4. Furthermore, the strong evidence for a field reversal between the local and Sagittarius-Carina arms in Q1 suggests the reversal must slice through the Sagittarius-Carina arm if this reversal is continuous between Q1 and Q4, such as in the model proposed by Vallée (2005). The subsequently implied lack of alignment of the magnetic field with the Sagittarius-Carina arm is consistent with earlier observations of at least a $5^{\circ}$ offset in pitch angle between the orientation of the local field and that of the local spiral arm (Beck 2007).

\section{Modeling the Large-Scale Magnetic Field}

As a separate approach to qualitative observational analysis of the data, we can fit global magnetic field models to the RM data (with assumptions regarding the electron distribution in the Galaxy), to explore the structure of the uniform field and the location or existence of magnetic field reversals. Here we use the CL02 electron density model and the technique of Brown (2002) which uses linear inversion theory (Menke 1984) to obtain a least-squares fit to the data. With this method, the boundaries of magnetic field regions are fixed a priori but the strength and direction of the field within these regions are not. The model is fit to the observed RMs to derive the strength and direction within each region. This contrasts previous analyses which also employed separate models for the electron density and magnetic field, but where the direction of the field was an input to the model (e.g. Indrani \& Despande 1998).

We present here a simple model where we consider nine magnetic field regions, eight of which are either arm or interarm regions, and the ninth corresponds to the molecular ring. This model does not include the differing pitch angle between the magnetic and spiral arms discussed in section 3. but is instead designed to be directly comparable with the results of Weisberg et al. (2004) and Han et al. (2006). The region boundaries are delineated by the green spirals in Figure 4 . The constraints for the model field within each arm or interarm region are: 1) a log-spiral with a pitch angle of $11.5^{\circ}$ as shown; 2) a field strength that is inversely proportional to Galactic radius (eg. Heiles 1996b; Beck 2001; Brown et al. 2003a); 3) a zero vertical component; 4) a coherent direction within each region. We set the magnetic field within the molecular ring to have the same constraints as in the arms, except that the field is assumed to be purely azimuthal. At Galactic radii less than $3 \mathrm{kpc}$ or greater than $20 \mathrm{kpc}$, or at more than $1 \mathrm{kpc}$ from the mid-plane, the field is set to zero.

We constrain the model using RM data from individual sources only within the SGPS region (120 pulsars, 148 SGPS EGS, and 1 EGS from Broten et. al 1988; see sections 2 and 3) 1 For modeling purposes, we assume that the intergalactic contribution to the EGS RMs is negligible (Simard-Normandin \& Kronberg 1980; Gaensler et al. 2005), so that the EGS may be considered to reside at Galactocentric radii of 20 kpc. Most of the published pulsar distances used are based on the CL02 model, and are therefore consistent with this model. As a consequence of the limited sky coverage of the RM data used here, we confine our analysis of the result to within the SGPS longitudes.

The best-fit output for this model is a CW field everywhere except for a CCW field in the ScutumCrux arm and in the molecular ring, as shown in

\footnotetext{
${ }^{1}$ Global models using data from all quadrants is beyond the scope of this paper, but will be presented in a future paper.
} 
Figure 4. Figure 5 shows a plot of both the measured and modeled RMs for the individual SGPS EGS data (top panel), these data averaged and smoothed as in the middle panel of Figure 3 (middle panel), and the measured and modeled RMs for individual pulsars (bottom panel).

This model is able to closely reproduce the RM structure seen in the smoothed SGPS EGS data (recall that the fit is to the individual EGS and pulsar data) . However, there are two (relatively small) discrepancies between the model and the observed data towards the outer Galaxy. The first is in the vicinity around $\ell \sim 275 \mathrm{deg}$, where the measured data are more negative than the modeled data as also seen in the top panel Figure 5. In Figure 2, there is a contained region of small, negative $\mathrm{RM}$ between $270^{\circ}<l<283^{\circ}$ at $b>0^{\circ}$, resembling a magnetic bubble like that discussed by Clegg et al. (1992) and Brown \& Taylor (2001). RMs at these longitudes nominally should be dominated by the field in the local arm. Thus, if the negative RMs seen here were to be attributed to the large-scale field, this would imply that the field is directed counter-clockwise in the local arm. This is contrary to the many studies that show the field is clockwise in the local arm, as discussed in section 1. Interestingly, this region was previously identified in the Parkes 2.4-GHz survey as containing a polarized feature of unknown origin (Duncan et al. 1997). Since the lines-of-sight in the outer Galaxy are considerably smaller through the interstellar medium, compared to lines-of-sight through the inner Galaxy, it is likely that this localized feature is dominating the RM for these EGS. As a result, averaging the negative RMs through this localized feature with the otherwise positive RMs of the local arm creates the effect of $\overline{\mathrm{RM}} \sim 0$ at $l \sim 275^{\circ}$.

The other discrepancy is around $\ell \sim 265^{\circ}$ where the measured RMs are more positive than the modeled RMs. Lines-of-sight at this longitude are again through the outer Galaxy where localized features including the Gum nebula (Chanot \& Sivan 1983) could dominate the RM. As seen in the top panel of Figure 3 there are two EGS sources at $\ell=263^{\circ}$ that have RMs roughly double that of neighboring sources which biases the average RM near this longitude. Interestingly, there is also a localized peak in the pulsar data near this longitude, as seen in the bottom panel of Figure 3. The model tries to fit both the negative region around $\ell \sim 275^{\circ}$ and the more positive region around $\ell \sim 265^{\circ}$, with the result being a compromise between the two.

Although the individual pulsar data are noisier than the averaged EGS data, the trends of the pulsar data are also reproduced by this model. In particular, the model supports our observational conclusion that the Q4-component of the Sagittarius-Carina arm is directed CW, while the Q4-component of the Scutum-Crux arm is directed CCW.

Finally, we note that the direction of the Norma arm field is not well constrained by this model or by the data. Regardless of whether the field in the Norma arm is oriented CW or CCW, the results from this model contrast the previous suggestions of reversals with every arm (Weisberg et al. 2004) or at every arm-interarm boundary (Han et al. 2006).

\section{Summary}

We present the rotation measures for 148 extragalactic sources found in the southern Galactic plane survey. The oscillations of rotation measure with longitude revealed by these sources, and as also seen in pulsar RM data, highlight the dominating effect of the spiral arms on rotation measure. Both empirically and with a direct fit to measurements, the new data show conclusively that the field is directed clockwise in the fourth-quadrant component of the SagittariusCarina arm, and that a field reversal exists between the Sagittarius-Carina arm and the ScutumCrux arms in the fourth quadrant.

A definitive measurement of the number of large-scale magnetic reversals in the Galaxy can only emerge from an analysis that includes pulsar and EGS RM data at all Galactic longitudes, and which considers a wide range of distinct field configurations. In addition, the technique presented here is constrained to geometries imposed by the CL02 model. With these caveats, the results from our study of southern RMs indicate that far fewer magnetic reversals are needed to explain the data than other recent studies have suggested. 
We thank the anonymous referee for the insightful comments that have improved this manuscript. The Australia Telescope is funded by the Commonwealth of Australia for operation as a National Facility managed by CSIRO. This work was facilitated in part by an associateship grant to JCB by the Alberta Ingenuity Fund. MH acknowledges support from the National Radio Astronomy Observatory, which is operated by Associated Universities, Inc., under cooperative agreement with the NSF. BMG and MH acknowledge the support of the NSF through grant AST-0307358.

\section{REFERENCES}

Beck, R. 2001, Space Sci. Rev., 99, 243

Beck, R. 2007 in Polarisation 2005, F. Boulanger \& M. A. Miville-Deschenes (eds.), EAS Publication Series, 19 (astro-ph/0603531)

Brown, J. C. 2002, PhD Thesis, University of Calgary

Brown, J. C., Taylor, A. R. 2001, ApJ, 563, L31

Brown, J. C., Taylor, A. R., Wielebinski, R., \& Mueller, P. 2003, ApJ, 592, L29

Brown, J. C., Taylor, A. R., \& Jackel, B. J. 2003b, ApJS, 145, 213

Broten, N. W., MacLeod, J. M., \& Vallée, J. P. 1988, ApSS, 141, 303

Caswell, J. L., McClure-Griffiths, \& N. M., Cheung, M. C. M. 2004, MNRAS, 352, 1405

Clegg, A. W., Cordes, J. M., Simonetti, J. M., \& Kulkarni, S. R. 1992, ApJ, 386, 143

Cordes, J. M., \& Lazio, T. J. W. 2002, preprint (astro-ph/0207156) CL02

Chanot, A. \& Sivan, J. P. 1983, A\&A, 121, 19

Duncan, A. R., Haynes, R. F., Jones, K. L., \& Stewart, R. T. 1997, MNRAS, 291, 279

Frick, P., Stepanov, R., Shukurov, A., \& Sokoloff, D. 2001, MNRAS, 325, 649

Gaensler, B. M., Dickey, J. M., McClure-Griffiths, N. M., Green, A. J., Wieringa, M. H., \& Haynes, R. F. 2001, ApJ, 549, 959
Gaensler, B. M., Haverkorn, M., Staveley-Smith, L., Dickey, J. M., McClure-Griffiths, N. M., Dickel, J. R. \& Wolleben, M. 2005, Science, 307, 1610

Han, J. L., Manchester, R. N., \& Qiao, G. J. 1999, MNRAS, 306, 371

Han, J. L., Manchester, R. N., Lyne, A. G., Qiao, G. J., \& van Straten, W., 2006, ApJ, 642, 868

Haverkorn, M., Gaensler, B. M., Brown, J. C., Bizunok, N. S., McClure-Griffiths, N. M., Dickey, J. M., \& Green, A. J. 2006a, ApJ, L33

Haverkorn, M., Gaensler, B. M., McClureGriffiths, N. M., Dickey, J. M., \& Green, A. J. 2006b, ApJS, 167, 230

Heiles, C. 1996a, ApJ, 462, 316

Heiles, C. 1996b, in Polarimetry of the Interstellar Medium, W. Roberge \& D. Whittet (eds), 97 (ASPCS), 457

Indrani, C. \& Deshpande, A. A. 1998, New Astronomy, 4, 33

Lyne, A. G. \& Smith, F. G. 1989, MNRAS, 237, 533

Manchester, R. N. 1972, ApJ, 172, 43

Manchester, R. N. 1974, ApJ, 188, 637

McClure-Griffiths, N. M., Dickey, J. M., Gaensler, B. M., Green, A. J., Haverkorn, M., \& Strasser, S. 2005, ApJS, 158, 178

Menke, W. 1984, Geophysical Data Analysis: Discrete Inverse Theory (Academic Press, inc.)

Mitra, D., Wielebinski, R., Kramer, M., \& Jessner, A. $2003,403,585$

Rand, R. J. \& Lyne, A.G. 1994, MNRAS, 268, 497

Shukurov, A. 2005 in Cosmic Magnetic Fields, R. Wielebinski \& R. Beck (eds.), Springer LNP 664,113

Simard-Normandin, M. \& Kronberg, P.P. 1980, ApJ, 242, 74

Taylor, A. R., Gibson,S. J., Peracaula, M. et al. 2003, AJ, 125, 3145 
Taylor, J. H., Manchester, R. N., \& Lyne, A. G. 2000, VizieR Online Data Catalog, 7189

Thomson, R. C., Nelson \& A. H. 1980, MNRAS, 191,863

Vallée, J. P. 1980, A\&A, 86, 251

Vallée, J. P. 2005, ApJ, 619, 297

Weisberg, J. M., Cordes, J. M., Kuan, B., Devine, K. E., Green, J. T., \& Backer, D. C. 2004, ApJS, 150, 317

This 2-column preprint was prepared with the AAS LATEX macros v5.2. 
TABLE 1

Rotation Measures of the SGPS

\begin{tabular}{|c|c|c|c|c|c|c|}
\hline $\begin{array}{l}\ell^{a} \\
\left(^{\circ}\right)\end{array}$ & $\begin{array}{l}b^{a} \\
\left(^{\circ}\right)\end{array}$ & $\begin{array}{c}\alpha \\
(\mathrm{J} 2000)\end{array}$ & $\begin{array}{c}\delta \\
(\mathrm{J} 2000)\end{array}$ & $\begin{array}{c}I^{b} \\
(\mathrm{mJy})\end{array}$ & $\begin{array}{l}m^{c} \\
(\%)\end{array}$ & $\begin{array}{c}\mathrm{RM} \\
\left(\operatorname{rad~m}^{-2}\right)\end{array}$ \\
\hline 253.30 & 0.89 & 081946.2 & -344205 & 179 & 5.8 & $-8 \pm 12$ \\
\hline 253.52 & 0.83 & 082008.4 & -345520 & 21 & 6.4 & $-50 \pm 26$ \\
\hline 253.68 & -0.60 & 081447.2 & -355108 & 72 & 3.5 & $-349 \pm 27$ \\
\hline 254.16 & -0.34 & 081710.3 & -360601 & 64 & 5.5 & $-338 \pm 19$ \\
\hline 254.60 & -0.87 & 081612.7 & -364605 & 62 & 4.3 & $-15 \pm 24$ \\
\hline 254.81 & 0.93 & 082408.9 & -355521 & 87 & 13.7 & $+84 \pm 13$ \\
\hline 254.95 & 0.63 & 082320.7 & -361156 & 41 & 4.9 & $+8 \pm 32$ \\
\hline 255.16 & 0.24 & 082221.9 & -363603 & 92 & 5.3 & $-35 \pm 16$ \\
\hline 255.27 & 0.16 & 082220.2 & -364353 & 84 & 3.4 & $+26 \pm 23$ \\
\hline 255.36 & -0.26 & 082052.1 & -370248 & 136 & 9.0 & $+97 \pm 10$ \\
\hline 255.36 & 0.50 & 082359.5 & -363700 & 94 & 8.0 & $-115 \pm 13$ \\
\hline 256.14 & 0.25 & 082511.9 & -372400 & 92 & 5.3 & $+44 \pm 17$ \\
\hline 256.64 & -0.22 & 082443.7 & -380440 & 104 & 4.7 & $+172 \pm 15$ \\
\hline 257.47 & 0.54 & 083020.4 & -381827 & 46 & 9.5 & $+23 \pm 20$ \\
\hline 257.71 & -0.66 & 082602.2 & -391213 & 117 & 4.0 & $+144 \pm 20$ \\
\hline 257.92 & 0.65 & 083207.7 & -383633 & 383 & 2.3 & $+76 \pm 14$ \\
\hline 258.52 & 1.02 & 083530.1 & -385223 & 118 & 2.7 & $+196 \pm 26$ \\
\hline 258.77 & 0.08 & 083223.4 & -393738 & 131 & 3.4 & $+221 \pm 16$ \\
\hline 259.05 & -0.72 & 082949.9 & -401935 & 123 & 5.7 & $+175 \pm 12$ \\
\hline 259.05 & -0.75 & 082945.1 & -402052 & 103 & 10.0 & $+150 \pm 12$ \\
\hline 259.77 & 1.22 & 084015.2 & -394439 & 33 & 5.8 & $+250 \pm 29$ \\
\hline 260.41 & -0.43 & 083521.8 & -411450 & 101 & 3.0 & $+221 \pm 18$ \\
\hline 260.52 & -0.55 & 083511.4 & -412444 & 25 & 5.8 & $+247 \pm 31$ \\
\hline 260.69 & -0.23 & 083705.4 & -412108 & 224 & 3.2 & $+204 \pm 12$ \\
\hline 263.20 & 1.07 & 085056.2 & -423054 & 165 & 5.6 & $+739 \pm 14$ \\
\hline 263.22 & 1.08 & 085102.6 & -423132 & 164 & 4.4 & $+826 \pm 19$ \\
\hline 263.50 & 0.17 & 084812.7 & -431914 & 139 & 0.9 & $+260 \pm 28$ \\
\hline 264.24 & 0.88 & 085348.3 & -432559 & 112 & 11.0 & $+406 \pm 9$ \\
\hline 265.69 & 0.85 & 085854.5 & -443341 & 106 & 1.8 & $+70 \pm 28$ \\
\hline 266.14 & 1.08 & 090133.6 & -444449 & 66 & 3.2 & $+211 \pm 29$ \\
\hline 266.27 & 0.66 & 090015.2 & -450717 & 95 & 3.4 & $+396 \pm 18$ \\
\hline 267.03 & 0.04 & 090027.1 & -460615 & 58 & 3.8 & $+298 \pm 21$ \\
\hline 267.17 & 0.47 & 090250.7 & -455536 & 598 & 0.3 & $+323 \pm 28$ \\
\hline 268.62 & 0.58 & 090856.5 & -465553 & 231 & 2.1 & $+256 \pm 15$ \\
\hline 269.05 & 0.17 & 090854.5 & -473115 & 118 & 3.6 & $+456 \pm 16$ \\
\hline 269.55 & 0.45 & 091209.7 & -474135 & 156 & 1.9 & $+137 \pm 18$ \\
\hline 270.56 & -0.85 & 091032.9 & -491914 & 58 & 5.5 & $-152 \pm 23$ \\
\hline 270.91 & 0.93 & 091952.0 & -482010 & 57 & 6.2 & $-149 \pm 22$ \\
\hline 271.22 & -0.35 & 091537.0 & -492704 & 66 & 2.5 & $+215 \pm 29$ \\
\hline 271.30 & -0.06 & 091712.3 & -491817 & 83 & 5.0 & $+136 \pm 16$ \\
\hline
\end{tabular}


TABLE 1-Continued

\begin{tabular}{|c|c|c|c|c|c|c|}
\hline $\begin{array}{l}\ell^{a} \\
\left(^{\circ}\right)\end{array}$ & $\begin{array}{l}b^{a} \\
\left(^{\circ}\right)\end{array}$ & $\begin{array}{c}\alpha \\
(\mathrm{J} 2000)\end{array}$ & $\begin{array}{c}\delta \\
(\mathrm{J} 2000)\end{array}$ & $\begin{array}{c}I^{b} \\
(\mathrm{mJy})\end{array}$ & $\begin{array}{l}m^{c} \\
(\%)\end{array}$ & $\begin{array}{c}\mathrm{RM} \\
\left(\operatorname{rad~m^{-2}}\right)\end{array}$ \\
\hline 271.52 & -1.01 & 091354.3 & -500734 & 120 & 1.7 & $+75 \pm 28$ \\
\hline 271.70 & -0.38 & 091730.7 & -494916 & 116 & 3.5 & $-93 \pm 18$ \\
\hline 272.36 & 0.62 & 092445.8 & -493444 & 21 & 5.6 & $-296 \pm 28$ \\
\hline 273.46 & 0.68 & 092958.1 & $\begin{array}{lll}-50 & 17 & 41\end{array}$ & 18 & 13.6 & $-106 \pm 19$ \\
\hline 273.57 & 1.28 & 093300.8 & -495547 & 229 & 1.9 & $+20 \pm 25$ \\
\hline 274.77 & 0.25 & 093414.3 & -513018 & 51 & 3.9 & $-76 \pm 22$ \\
\hline 275.02 & 0.82 & 093751.7 & -511507 & 26 & 8.4 & $-101 \pm 25$ \\
\hline 275.48 & -0.68 & 093332.0 & -524018 & 282 & 2.8 & $+248 \pm 13$ \\
\hline 275.56 & 1.02 & 094117.5 & -512726 & 281 & 6.3 & $+16 \pm 7$ \\
\hline 275.56 & -0.20 & 093603.4 & -522203 & 46 & 2.9 & $+112 \pm 30$ \\
\hline 275.83 & 0.16 & 093855.3 & -521641 & 170 & 9.3 & $-107 \pm 7$ \\
\hline 275.86 & 0.94 & 094225.0 & -514248 & 29 & 11.0 & $0 \pm 21$ \\
\hline 276.46 & 0.89 & 094509.7 & -520829 & 153 & 2.5 & $-17 \pm 20$ \\
\hline 277.44 & 0.86 & 094958.5 & 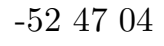 & 10 & 12.1 & $-71 \pm 28$ \\
\hline 277.78 & -0.73 & 094452.1 & -541345 & 127 & 2.0 & $-36 \pm 23$ \\
\hline 277.78 & -0.81 & 094432.3 & -541732 & 690 & 7.3 & $-77 \pm 3$ \\
\hline 278.04 & 0.75 & 095237.5 & -531508 & 635 & 7.8 & $-104 \pm 3$ \\
\hline 278.37 & 0.15 & 095147.9 & -535546 & 18 & 13.7 & $-4 \pm 24$ \\
\hline 278.43 & 0.53 & 095342.8 & -534012 & 42 & 4.4 & $+20 \pm 27$ \\
\hline 278.47 & -0.30 & 095022.2 & -542021 & 22 & 6.6 & $-116 \pm 24$ \\
\hline 279.04 & -0.88 & 095055.5 & -550904 & 242 & 2.4 & $+239 \pm 17$ \\
\hline 279.09 & 0.89 & 095846.2 & -534721 & 47 & 5.0 & $-120 \pm 25$ \\
\hline 279.15 & -0.63 & 095236.5 & -550108 & 36 & 11.2 & $+341 \pm 24$ \\
\hline 279.15 & -0.65 & 095232.6 & -550237 & 44 & 6.2 & $+329 \pm 20$ \\
\hline 279.33 & 0.80 & 095941.3 & -540011 & 35 & 7.8 & $-157 \pm 18$ \\
\hline 279.80 & 1.21 & 100353.7 & -535724 & 25 & 9.7 & $-145 \pm 25$ \\
\hline 280.53 & 0.81 & 100618.9 & -544257 & 37 & 5.4 & $-10 \pm 23$ \\
\hline 280.62 & -0.14 & 100253.8 & -553209 & 134 & 3.7 & $-112 \pm 15$ \\
\hline 282.07 & -0.78 & 100836.2 & -565409 & 723 & 0.9 & $+862 \pm 16$ \\
\hline 282.46 & 0.24 & 101509.3 & -561728 & 109 & 2.0 & $+256 \pm 23$ \\
\hline 284.30 & 0.81 & 102839.3 & -564809 & 80 & 2.4 & $-547 \pm 25$ \\
\hline 285.15 & 0.96 & 103433.2 & $\begin{array}{llll}-57 & 06 & 17\end{array}$ & 268 & 0.9 & $+168 \pm 31$ \\
\hline 285.60 & 0.62 & 103612.7 & -573738 & 44 & 3.3 & $+368 \pm 35$ \\
\hline 286.04 & -1.05 & 103243.2 & -591754 & 2826 & 0.4 & $+809 \pm 14$ \\
\hline 286.89 & 0.59 & 104437.5 & -581643 & 58 & 3.0 & $+324 \pm 28$ \\
\hline 288.27 & -0.70 & 104934.1 & -600321 & 255 & 0.6 & $+491 \pm 25$ \\
\hline 290.81 & 0.74 & 111245.4 & -594727 & 1858 & 0.4 & $+419 \pm 23$ \\
\hline 292.90 & -0.02 & 112631.6 & -611337 & 52 & 6.6 & $+349 \pm 28$ \\
\hline 293.39 & 0.73 & 113219.4 & -604022 & 67 & 5.0 & $+121 \pm 20$ \\
\hline 293.73 & 0.63 & 113443.2 & -605206 & 30 & 3.4 & $+116 \pm 23$ \\
\hline
\end{tabular}


TABLE 1-Continued

\begin{tabular}{|c|c|c|c|c|c|c|}
\hline $\begin{array}{l}\ell^{a} \\
\left(^{\circ}\right)\end{array}$ & $\begin{array}{l}b^{a} \\
\left(^{\circ}\right)\end{array}$ & $\begin{array}{c}\alpha \\
(\mathrm{J} 2000)\end{array}$ & $\begin{array}{c}\delta \\
(\mathrm{J} 2000)\end{array}$ & $\begin{array}{c}I^{b} \\
(\mathrm{mJy})\end{array}$ & $\begin{array}{l}m^{c} \\
(\%)\end{array}$ & $\begin{array}{c}\mathrm{RM} \\
\left(\operatorname{rad~m^{-2}}\right)\end{array}$ \\
\hline 294.29 & -0.90 & 113531.6 & -622913 & 32 & 4.2 & $+449 \pm 26$ \\
\hline 294.38 & -0.75 & 113637.4 & -622207 & 265 & 0.7 & $+470 \pm 24$ \\
\hline 295.17 & 0.01 & 114455.1 & -615126 & 57 & 3.5 & $+363 \pm 22$ \\
\hline 295.23 & -1.05 & 114302.3 & -625356 & 114 & 2.1 & $-207 \pm 22$ \\
\hline 295.29 & -1.23 & 114305.7 & -630455 & 226 & 1.6 & $-43 \pm 29$ \\
\hline 296.18 & -0.59 & 115205.2 & -624059 & 118 & 3.7 & $+752 \pm 14$ \\
\hline 296.90 & 0.14 & 115931.2 & -620715 & 452 & 1.6 & $+1113 \pm 11$ \\
\hline 297.67 & 0.77 & 120652.0 & -613854 & 283 & 2.6 & $+570 \pm 11$ \\
\hline 299.42 & -0.23 & 122029.8 & -625337 & 55 & 4.0 & $+535 \pm 30$ \\
\hline 299.51 & -1.10 & 122024.8 & -634610 & 192 & 2.9 & $+315 \pm 21$ \\
\hline 300.25 & -0.01 & 122757.6 & -624542 & 109 & 4.1 & $+123 \pm 14$ \\
\hline 300.47 & -0.99 & 122906.5 & -634508 & 112 & 4.9 & $+412 \pm 18$ \\
\hline 300.65 & -0.41 & 123112.4 & -631140 & 809 & 0.9 & $+358 \pm 19$ \\
\hline 301.14 & -0.09 & 123539.6 & -625430 & 375 & 1.3 & $+350 \pm 18$ \\
\hline 301.70 & 0.25 & 124045.4 & -623601 & 21 & 5.2 & $+296 \pm 33$ \\
\hline 302.60 & -1.17 & 124826.2 & -640226 & 162 & 1.7 & $+159 \pm 25$ \\
\hline 303.30 & 0.51 & 125436.3 & -622125 & 149 & 3.5 & $-370 \pm 14$ \\
\hline 304.53 & 1.00 & 130500.1 & -614931 & 32 & 6.1 & $+40 \pm 28$ \\
\hline 305.62 & -1.16 & 131552.5 & -635409 & 44 & 10.5 & $-61 \pm 28$ \\
\hline 306.87 & 0.02 & 132547.5 & -623515 & 215 & 1.0 & $-197 \pm 27$ \\
\hline 306.92 & -0.70 & 132700.7 & -631746 & 275 & 2.0 & $+52 \pm 16$ \\
\hline 307.20 & -0.84 & 132939.3 & -632347 & 41 & 8.1 & $+382 \pm 18$ \\
\hline 308.64 & -0.62 & 134156.1 & -625538 & 40 & 2.8 & $-133 \pm 27$ \\
\hline 308.73 & 0.07 & 134133.5 & -621406 & 92 & 1.4 & $-661 \pm 29$ \\
\hline 308.93 & 0.40 & 134241.5 & -615238 & 152 & 2.5 & $-752 \pm 17$ \\
\hline 309.06 & 0.84 & 134300.9 & -612452 & 670 & 2.3 & $-504 \pm 10$ \\
\hline 310.20 & -1.04 & 135609.7 & -625930 & 46 & 8.5 & $-584 \pm 19$ \\
\hline 312.37 & -0.04 & 141137.1 & -612554 & 129 & 2.3 & $-438 \pm 28$ \\
\hline 313.96 & -0.76 & 142614.9 & -613458 & 88 & 2.3 & $-480 \pm 22$ \\
\hline 313.99 & 0.94 & 142136.9 & -595901 & 507 & 1.8 & $-828 \pm 17$ \\
\hline 314.02 & 1.01 & 142140.3 & -595420 & 757 & 1.7 & $-579 \pm 20$ \\
\hline 314.50 & 0.30 & 142714.8 & -602407 & 86 & 5.3 & $-738 \pm 19$ \\
\hline 314.82 & 0.89 & 142757.3 & -594358 & 92 & 3.7 & $-507 \pm 25$ \\
\hline 316.64 & 1.15 & 144015.0 & -584730 & 64 & 4.6 & $-525 \pm 27$ \\
\hline 317.54 & -0.57 & 145227.0 & -595811 & 152 & 2.1 & $+395 \pm 27$ \\
\hline 318.53 & 0.30 & 145618.1 & -584429 & 326 & 1.0 & $+53 \pm 29$ \\
\hline 319.34 & 1.08 & 145858.6 & -574031 & 1101 & 0.7 & $+241 \pm 21$ \\
\hline 319.39 & 0.74 & 150028.7 & -575704 & 85 & 1.4 & $+279 \pm 30$ \\
\hline 320.83 & 0.88 & 150919.7 & -570719 & 454 & 0.8 & $-8 \pm 20$ \\
\hline 321.48 & 1.02 & 151254.7 & -564020 & 933 & 2.0 & $-243 \pm 8$ \\
\hline
\end{tabular}


TABLE 1 - Continued

\begin{tabular}{|c|c|c|c|c|c|c|}
\hline $\begin{array}{l}\ell^{a} \\
\left(^{\circ}\right)\end{array}$ & $\begin{array}{l}b^{a} \\
\left(^{\circ}\right)\end{array}$ & $\begin{array}{c}\alpha \\
(\mathrm{J} 2000)\end{array}$ & $\begin{array}{c}\delta \\
(\mathrm{J} 2000)\end{array}$ & $\begin{array}{c}I^{b} \\
(\mathrm{mJy})\end{array}$ & $\begin{array}{l}m^{c} \\
(\%)\end{array}$ & $\begin{array}{c}\mathrm{RM} \\
\left(\operatorname{rad~m}^{-2}\right)\end{array}$ \\
\hline 321.58 & -0.76 & 152029.3 & -580741 & 1831 & 1.1 & $-138 \pm 9$ \\
\hline 322.05 & -0.95 & 152417.5 & $\begin{array}{lll}-58 & 01 & 49\end{array}$ & 78 & 5.4 & $-397 \pm 17$ \\
\hline 323.15 & -0.52 & 152919.5 & -570349 & 47 & 2.9 & $+83 \pm 25$ \\
\hline 324.77 & 0.61 & 153410.5 & -551230 & 161 & 3.9 & $-66 \pm 14$ \\
\hline 325.81 & 1.08 & 153805.1 & -541308 & 608 & 0.6 & $-15 \pm 31^{d}$ \\
\hline 325.83 & -0.30 & 154357.7 & -551826 & 247 & 1.8 & $+356 \pm 18$ \\
\hline 326.69 & -1.16 & 155228.2 & -552704 & 95 & 9.3 & $-142 \pm 22$ \\
\hline 327.31 & 0.88 & 154701.4 & -532829 & 256 & 1.2 & $-189 \pm 26^{d}$ \\
\hline 328.36 & -0.41 & 155800.3 & -534830 & 493 & 0.3 & $-721 \pm 35$ \\
\hline 329.48 & 0.22 & 160057.1 & -523604 & 599 & 0.7 & $-100 \pm 25$ \\
\hline 330.12 & -1.08 & 160949.5 & -530833 & 218 & 3.0 & $-931 \pm 25$ \\
\hline 332.14 & 1.03 & 161010.8 & -501321 & 151 & 2.8 & $-754 \pm 22^{d}$ \\
\hline 333.72 & -0.27 & 162255.5 & -500331 & 108 & 2.5 & $+204 \pm 29$ \\
\hline 335.32 & 0.60 & 162600.4 & -481836 & 352 & 2.9 & $-138 \pm 11$ \\
\hline 337.06 & 0.85 & 163201.7 & -465239 & 100 & 2.5 & $-739 \pm 32$ \\
\hline 339.65 & -0.24 & 164644.6 & -454000 & 222 & 2.2 & $-398 \pm 19$ \\
\hline 342.16 & -0.74 & 165752.7 & -440249 & 264 & 2.2 & $+127 \pm 15$ \\
\hline 342.62 & -0.45 & 165815.4 & -433019 & 50 & 3.9 & $-913 \pm 30$ \\
\hline 343.29 & 0.60 & 165601.8 & -421958 & 102 & 4.7 & $-1035 \pm 16$ \\
\hline 345.22 & 0.68 & 170202.9 & -404550 & 39 & 12.6 & $+183 \pm 21$ \\
\hline 347.40 & -1.04 & 171609.8 & -400224 & 72 & 4.0 & $-524 \pm 36$ \\
\hline 349.65 & -0.36 & 171958.3 & -374815 & 133 & 3.3 & $+110 \pm 20$ \\
\hline 350.52 & -0.73 & 172358.9 & -371818 & 127 & 2.2 & $+277 \pm 32$ \\
\hline 351.31 & -0.53 & 172522.9 & -363216 & 200 & 3.3 & $-247 \pm 15$ \\
\hline 351.82 & 0.17 & 172356.8 & -354334 & 118 & 6.1 & $+134 \pm 16$ \\
\hline 352.13 & 1.15 & 172051.7 & -345449 & 116 & 6.5 & $+76 \pm 30$ \\
\hline 355.43 & -0.81 & 173732.3 & -331440 & 76 & 4.2 & $+601 \pm 21$ \\
\hline 356.57 & 0.87 & 173345.9 & $\begin{array}{ll}-31 & 2237\end{array}$ & 116 & 1.6 & $+985 \pm 30$ \\
\hline
\end{tabular}

${ }^{a}$ The identified location is the peak of the gaussian fit to the source in polarised intensity. All sources were either unresolved or partially resolved.

${ }^{\mathrm{b}} I$ is the peak-pixel Stokes I value of the interferometric data.

${ }^{\mathrm{c}} m$ is the fractional polarisation (linear polarised intensity divided by Stokes I) averaged over the FWHM pixels used for RM calculation.

${ }^{\mathrm{d}}$ A RM for this source was calculated by Gaensler et al. (2001) using a simpler approach than the more rigorous method used here. These new values should replace the previously determined values. 


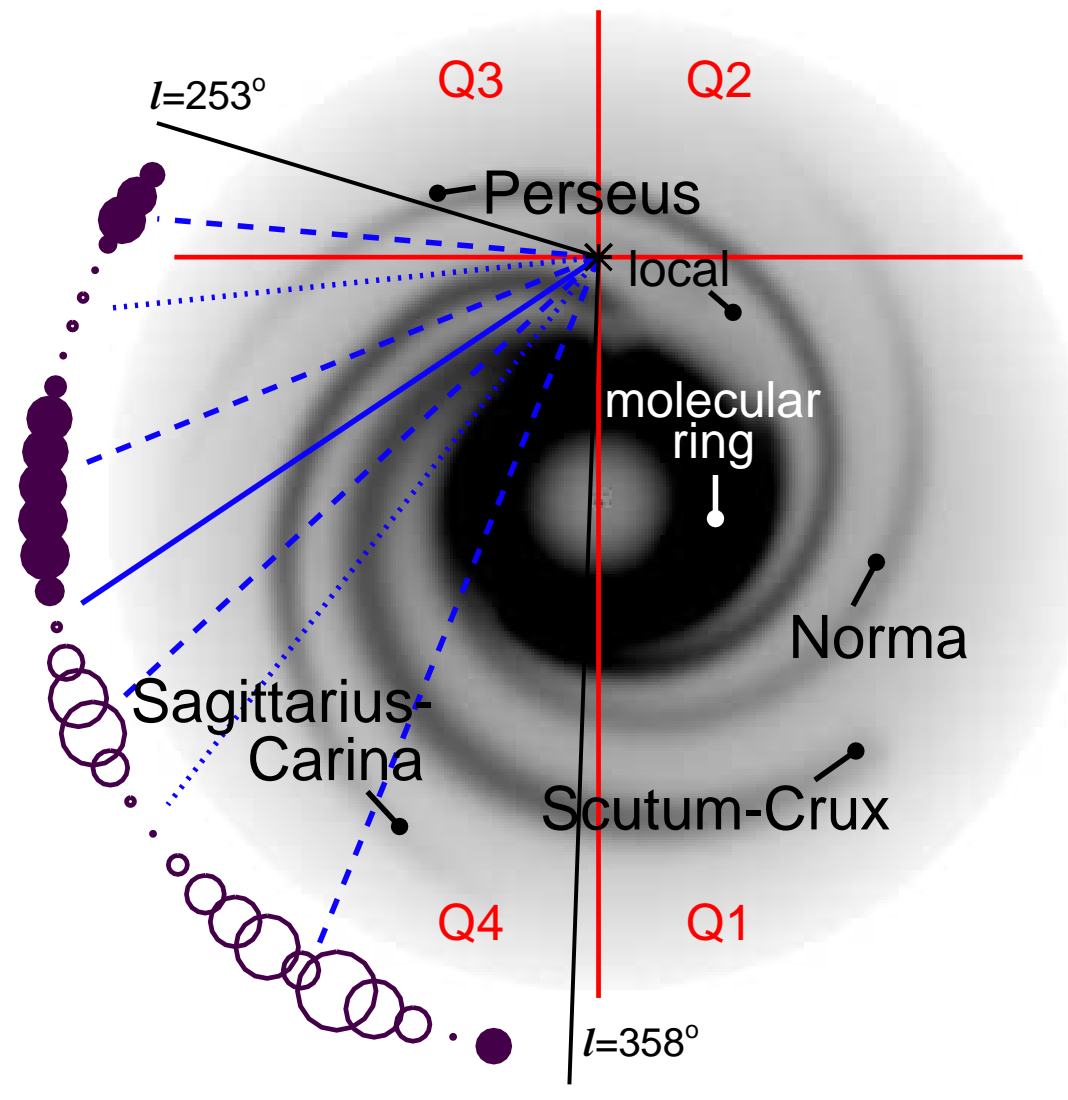

Fig. 1.- View of the Galaxy from above. The grey scale is the CL02 electron density model, with labels indicating the spiral arms, and the asterisk indicating the location of the Sun. Quadrants 1,2, 3, and 4 are labeled with Q1, Q2, Q3 and Q4, respectively. The bounding longitudes of the SGPS (Phase I) are indicated by black lines and are labeled. The circles represent the smoothed-averaged SGPS RMs as shown in the middle panel of Figure 3. Filled (open) circles indicate positive (negative) RM, with the size of the circles linearly proportional to $|\mathrm{RM}|$, truncated between 59 and $592 \mathrm{rad} \mathrm{m}^{-2}$ (the $|\mathrm{RM}|_{\max }$ from the middle panel of Figure 3) so that sources with $|\mathrm{RM}|<59 \mathrm{rad} \mathrm{m}^{-2}$ are set to $59 \mathrm{rad} \mathrm{m}^{-2}$. The blue dashed (dotted) lines are also from the middle panel of Figure 3, and indicate the approximate longitudes of $|\mathrm{RM}|$ maxima (minima) in the SGPS RM data. The solid blue line is the longitude where the RMs transition from primarily positive to primarily negative $\left(\ell \sim 304^{\circ}\right)$. 


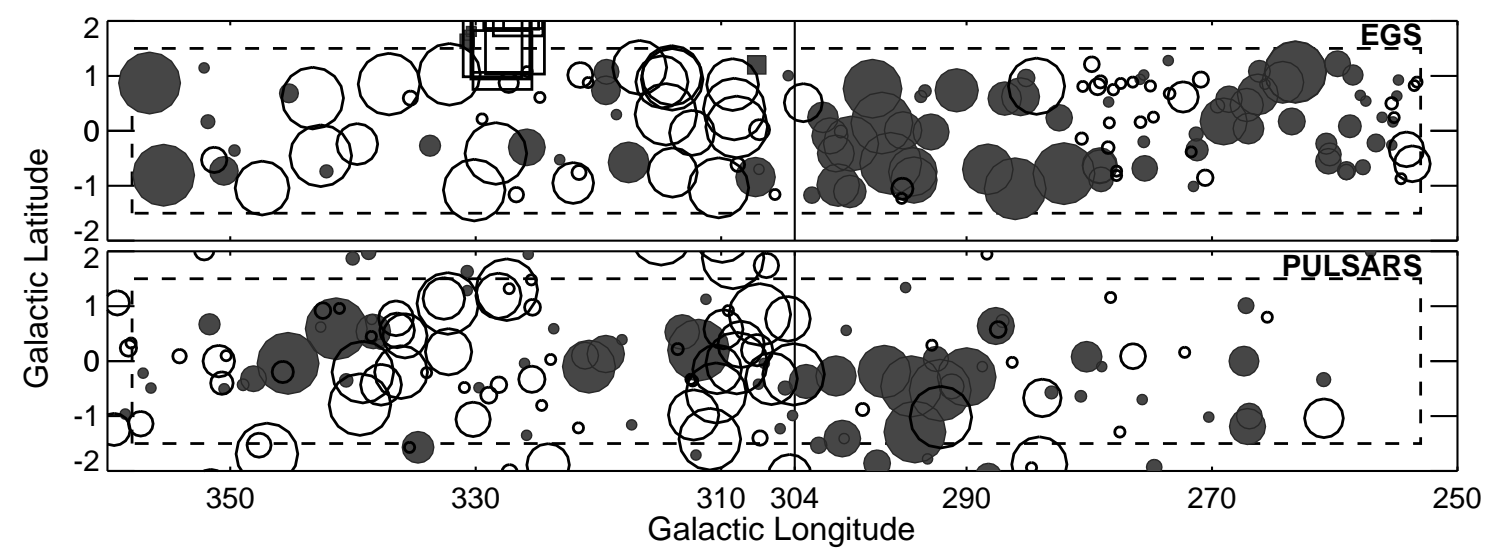

Fig. 2.- Rotation measures for sources in the SGPS. Top panel: extragalactic RM sources; Bottom panel: pulsar RM sources. Grey filled symbols indicate positive rotation measure, black open symbols indicate negative rotation measures; sizes of symbols are linearly proportional to the magnitude of RM truncated between 100-600 $\mathrm{rad} \mathrm{m}^{-2}$, so that sources with $|\mathrm{RM}|<100 \mathrm{rad} \mathrm{m}^{-2}$ are set to $100 \mathrm{rad} \mathrm{m}^{-2}$, and those with $|\mathrm{RM}|>600 \mathrm{rad} \mathrm{m}^{-2}$ are set to $600 \mathrm{rad} \mathrm{m}^{-2}$. In the top panel, the square at $\ell=307.1^{\circ}, b=1.2^{\circ}$ represents the EGS RM previously observed by Broten et al. (1988), while the squares at $\ell \sim 330^{\circ}$ represent the EGS RMs of the SGPS test region. The circles in the top panel represent the SGPS EGS as given in Table 1. The vertical line at $\ell=304^{\circ}$ is the approximate longitude where the EGS RMs change sign. The dashed boxes indicate boundaries of the SGPS region. 


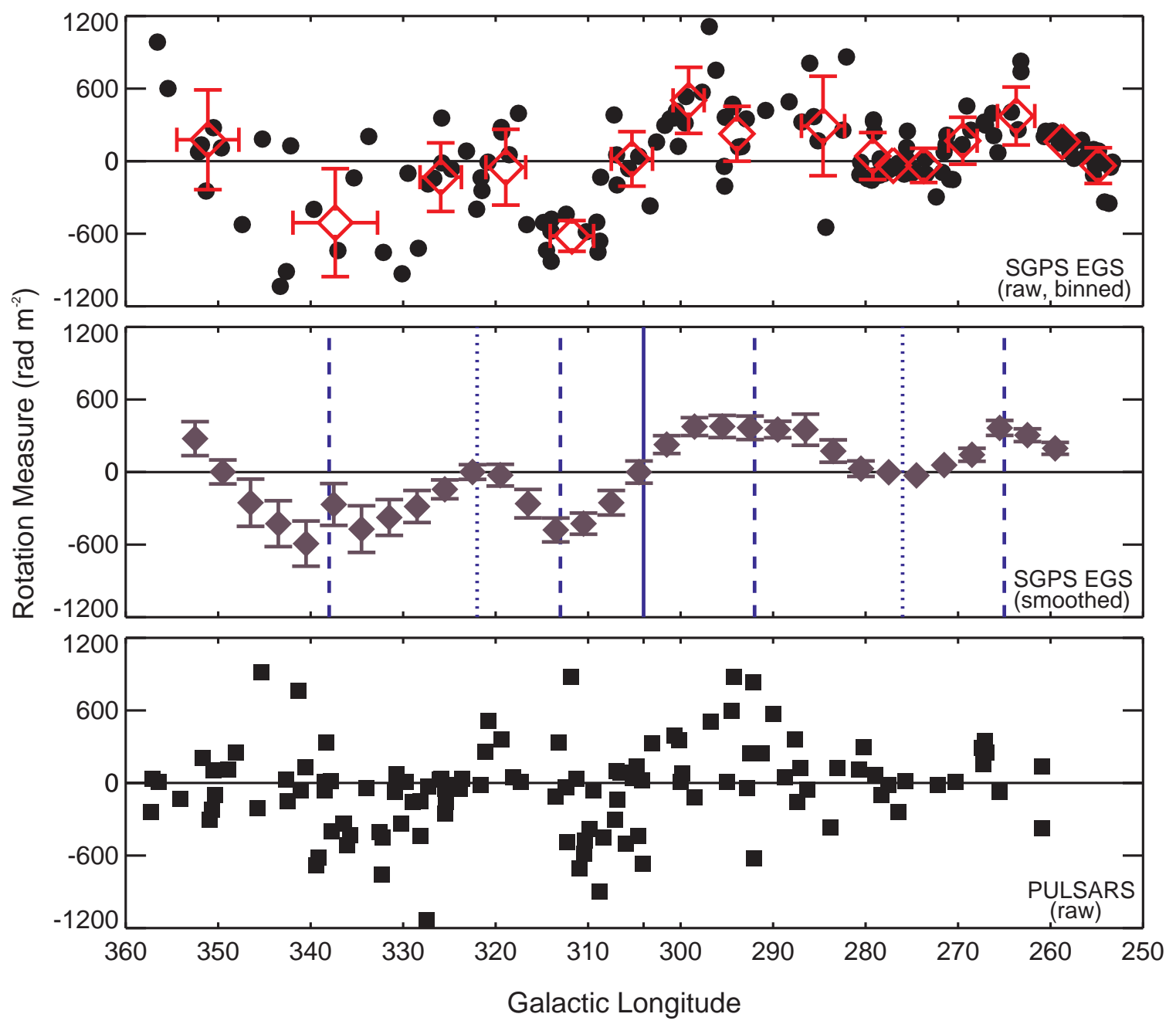

Fig. 3.- RM versus Galactic longitude for RM sources in the SGPS region. Top panel: circles represent the individual SGPS EGS sources (errors are smaller than the symbol size), while open red diamonds represent data averaged into into independent longitude bins containing 9 sources (the end bin at $255^{\circ}$ contains 13 sources). Where symbol size permits, the error bars are the standard deviation in longitude and RM for each bin. Middle panel: purple diamonds represent boxcar-averaged SGPS EGS data over 9 degrees in longitude with a stepsize of 3 degrees. In contrast to the binned data in the top panel, the error bars are the standard error of the mean. The solid line marks the approximate longitude of transition from predominantly positive RMs to negative RMs $\left(l \sim 304^{\circ}\right)$ Dotted lines (dashed lines) mark approximate longitudes of minimum (maximum) $|\mathrm{RM}|$ in SGPS data. Bottom panel: squares represent the individual pulsars with known RM in the SGPS region (errors are smaller than the symbol sizes). 


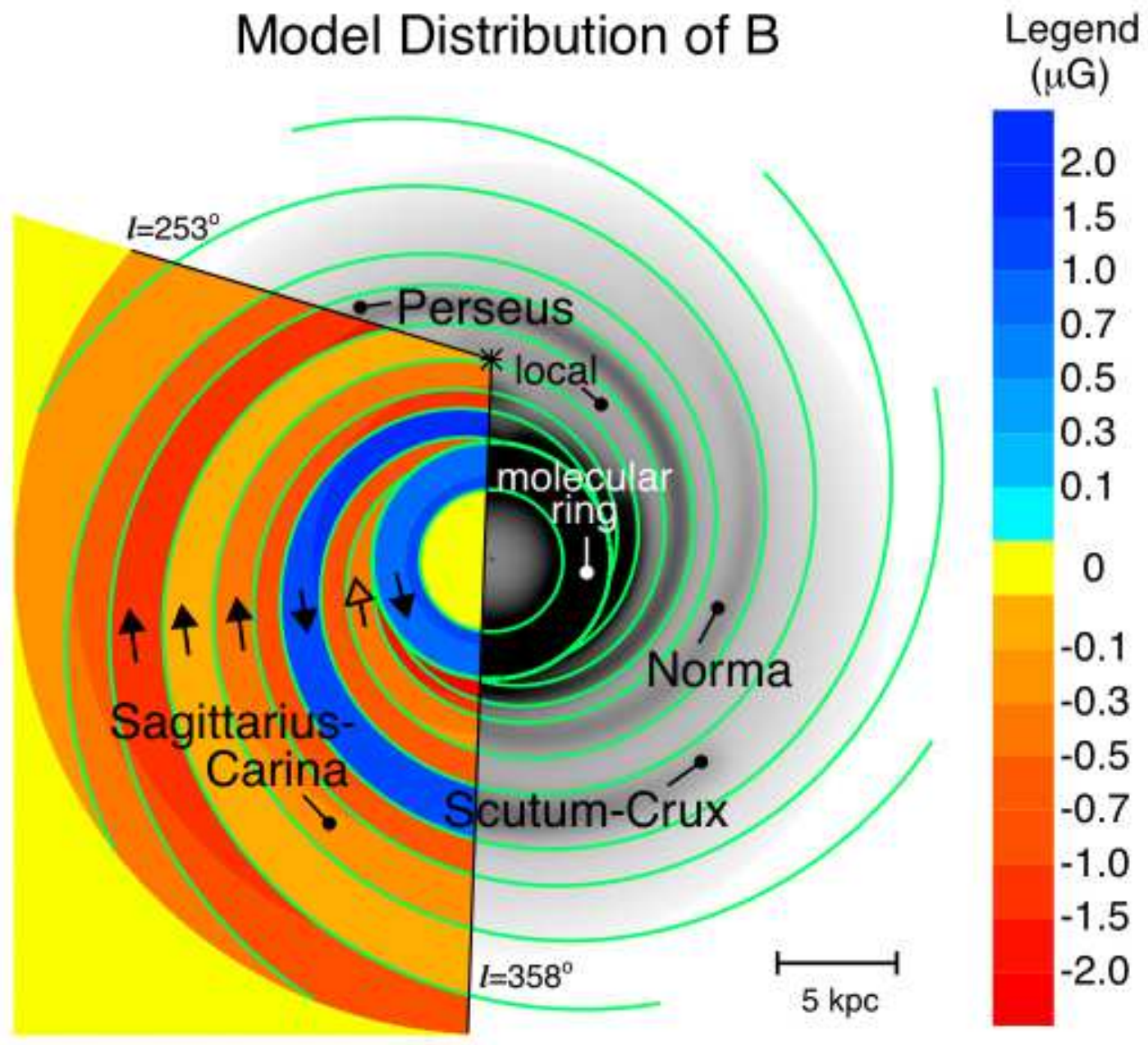

Fig. 4. - A simple model of the magnetic field in the SGPS region, constrained using individual RMs of 149 EGS and 120 pulsars. Colored regions indicate the total strength for the model fit of magnetic field corresponding to the regions identified by the green lines as discussed in the text. Red (blue) shading indicates a clockwise (counter-clockwise) field direction as viewed from the Galactic north pole. The direction of the field within the arms is also indicated by arrows. The open-head arrow on the Norma arm indicates the field direction in this arm is not well-constrained in this model configuration with the data used. The bounding longitudes of the SGPS (Phase I) are indicated by black lines and are labeled. The grey scale is the CL02 electron density model with labels indicating the spiral arms. 


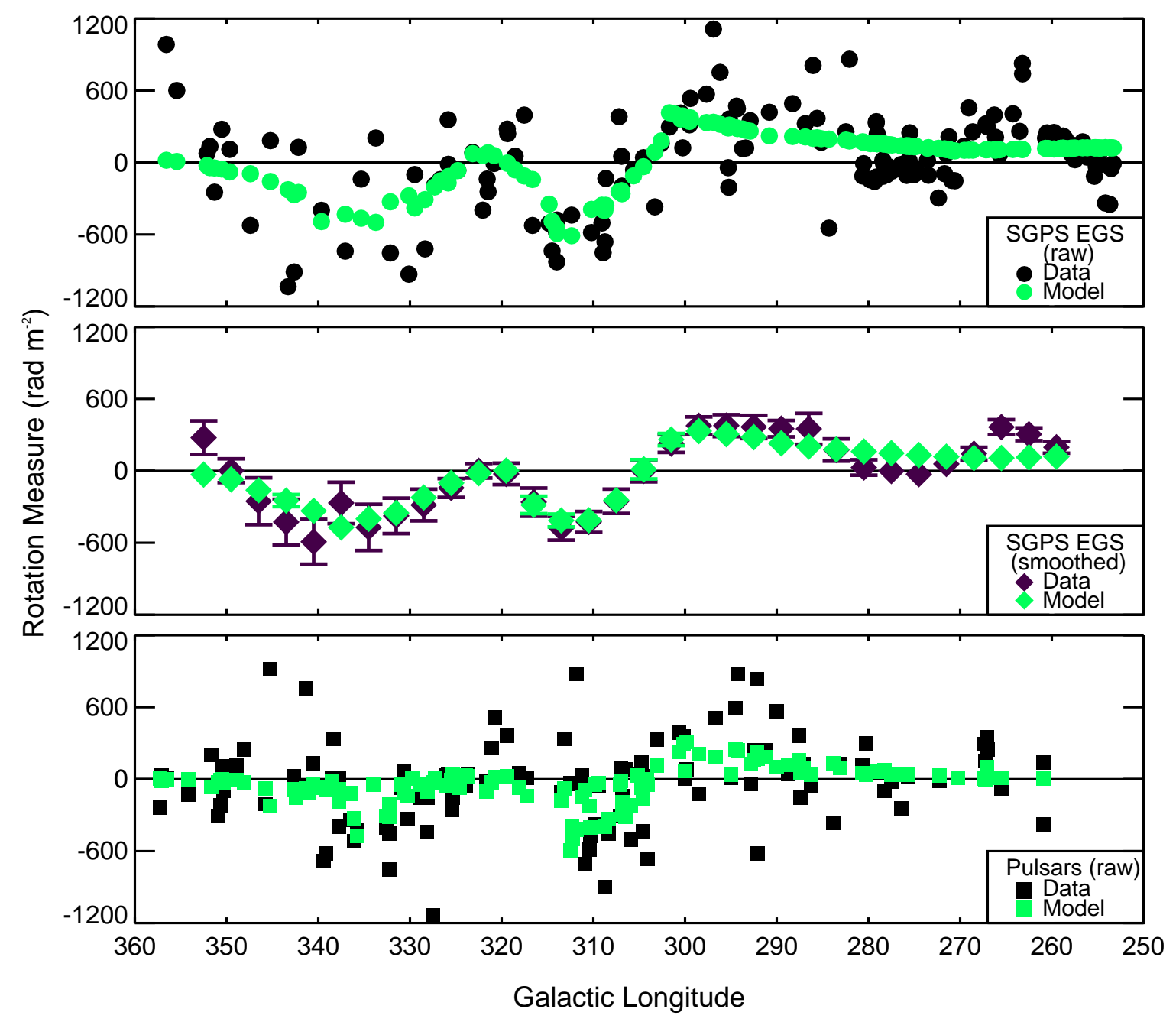

Fig. 5.- Comparison of modeled RMs (green symbols) and observed RMs. The modeled RMs are calculated using the CL02 electron density model and the magnetic field model shown in Figure 4 . The format of this figure follows that of Figure 3, where the individual SGPS EGS are shown in the top panel, smoothedaveraged SGPS EGS data are shown in the middle panel, and the individual pulsars are presented in the bottom panel. 